\title{
The role of the basal ganglia in implicit contextual learning: A study of Parkinson's disease
}

\author{
Marieke van Asselen ${ }^{\mathrm{a}, *}$, Inês Almeida ${ }^{\mathrm{a}}$, Rui Andre ${ }^{\mathrm{b}}$, Cristina Januário ${ }^{\mathrm{b}}$, \\ António Freire Gonçalves ${ }^{\mathrm{b}}$, Miguel Castelo-Branco ${ }^{\mathrm{a}}$ \\ a Visual Neuroscience Laboratory, IBILI, Coimbra, Portugal \\ ${ }^{\mathrm{b}}$ Department of Neurology, University Hospital Coimbra, Portugal
}

\section{A R T I C L E I N F O}

Article history:

Received 9 September 2008

Received in revised form 15 December 2008

Accepted 7 January 2009

Available online 16 January 2009

\section{Keywords:}

Implicit contextual cueing

Spatial

Memory

Parkinson's disease

Basal ganglia

\begin{abstract}
A B S T R A C T
Implicit contextual learning refers to the ability to memorize contextual information from our environment. This contextual information can then be used to guide our attention to a specific location. Although the medial temporal lobe is important for this type of learning, the basal ganglia might also be involved considering its role in many implicit learning processes. In order to understand the role of the basal ganglia in this top-down process, a group of non-demented early-stage Parkinson's patients were tested with a contextual cueing task. In this visual search task, subjects have to quickly locate a target among a number of distractors. To test implicit contextual learning, some of the configurations are repeated during the experiment, resulting in faster responses. A significant interaction effect was found between Group and Configuration, indicating that the control subjects responded faster when the spatial context was repeated, whereas Parkinson's patients failed to do so. These results, showing that the contextual cueing effect was significantly different for the patients than for the controls, suggest an important role for the basal ganglia in implicit contextual learning, thus extending previous findings of medial temporal lobe involvement. The basal ganglia are therefore not only involved in implicit motor learning, but may also have a role in purely visual implicit learning.
\end{abstract}

(C) 2009 Elsevier Ltd. All rights reserved.

\section{Introduction}

Our environment contains a large amount of information that is stable over time. For example, the positions of doors, windows, large pieces of furniture, trees and houses are usually fixed in relation to each other. Additionally, specific objects are found close to each other or in specific locations, for instance pots and pans are close to the stove in the kitchen. This type of visual organization can be memorized and can guide attention when scanning our environment (e.g. Chun \& Jiang, 1998). This mechanism is called implicit contextual learning and is thought to help internalize meaningful regularities and co-variations between objects and events in the visual world (Chun, 2000).

The contextual cueing effect can be studied by using a visual search task, in which a target $(\mathrm{T})$ has to be located among a number of distractors (L) (e.g. Chun \& Jiang, 1998). When the target is located, subjects have to indicate whether the target $\mathrm{T}$ is rotated to

\footnotetext{
* Corresponding author at: IBILI - Faculdade de Medicina, Azinhaga de Santa Comba - Celas, 3000-354 Coimbra, Portugal. Tel.: +351 239480220; fax: +351239480280 .

E-mail address: masselen@ibili.uc.pt (M. van Asselen).
}

the left or to the right. A large number of different configurations are generated, of which some are repeated throughout the experiment. Importantly, several studies have demonstrated that targets are located faster in trials with repeated configurations than with new configurations (e.g. Chun \& Jiang, 1998, 2003; Chun \& Phelps, 1999; Peterson \& Kramer, 2001). This effect can be obtained after 5 or 6 repetitions, indicating it is a fast effect, and can be found up to a week after encoding the information, indicating it remains in memory for a long time-period (Chun \& Jiang, 2003). Furthermore, subjects are not aware of the repeated contextual information and perform on change level during a Recognition memory task, indicating that this type of contextual learning is the result of an implicit memory process (e.g. Chun \& Jiang, 1998).

Although several studies have investigated the functional characteristics of implicit contextual learning (Chun \& Jiang, 1998; Chun and Jiang, 1999; Chun \& Jiang, 2003; Jiang \& Leung, 2005; Jiang, Song, \& Rigas, 2005; Olson \& Chun, 2002; Peterson \& Kramer, 2001; Tseng \& Li, 2004), little is known about its neural correlates. So far, research has mainly been aimed at defining the importance of the medial temporal lobes (MTL), including the hippocampus. Chun and Phelps (1999) found that amnesia patients with damage to the MTL were not able to benefit from repeated spatial context information, suggesting a crucial role for the MTL in implicit contextual 


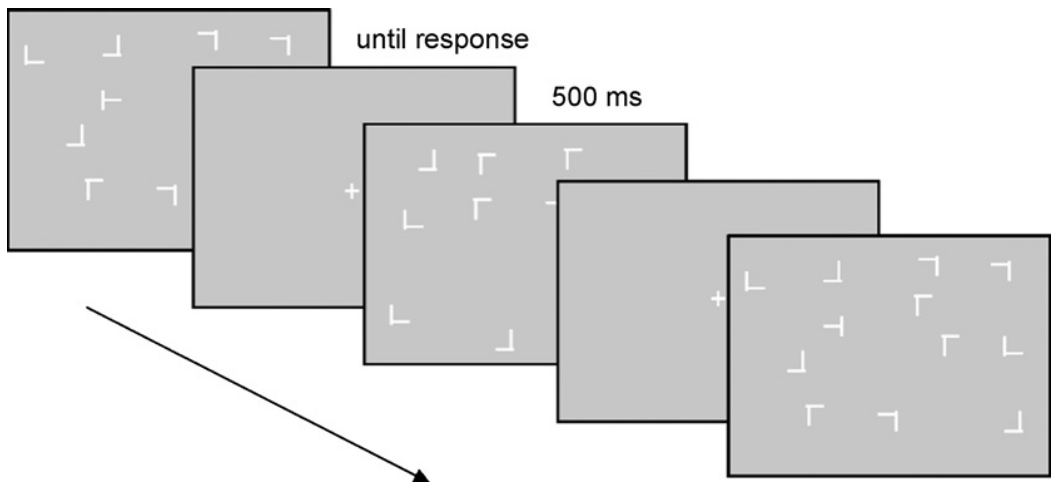

Fig. 1. Example of a few displays of the contextual cueing task.

learning. It was later suggested by Manns and Squire (2001) that the hippocampus per se is not essential and that an implicit contextual cueing impairment was only found after extended MTL damage. However, they could not indicate what MTL areas were essential. A recent brain imaging study did demonstrate the involvement of the hippocampus in this type of task (Greene, Gross, Elsinger, \& Rao, 2007).

Although the importance of MTL areas in implicit contextual learning has clearly been shown (Chun \& Phelps, 1999; Manns \& Squire, 2001), other brain areas are likely to be involved as well. Brain regions that are of great importance to many implicit learning and memory mechanisms are the basal ganglia. The involvement of these subcortical brain regions was demonstrated in humans by using a variety of paradigms, including the serial reaction time task and the probabilistic classification learning task. The serial reaction time task requires subjects to perform a series of finger movements that are typically shown on a computer screen. Unknown to the participants, the sequence of movements is repeated, resulting in faster response times than when a new sequence of movements is used. Patient (Kim et al., 2004; Wilkinson \& Jahanshahi, 2007) and neuroimaging studies using fMRI to define brain activity in healthy subjects (Daselaar, Rombouts, Veltman, Raaijmakers, \& Jonker, 2003; Rauch et al., 1997) have shown that the basal ganglia have an important role in this type of implicit learning (for a review see: Doyon, Penhune, \& Ungerleider, 2003). Probabilistic classification learning is another type of implicit memory that is often used to define characteristics and neural correlates of implicit learning. In a probabilistic classification learning task, different cues are probabilistically associated with one of two outcomes. Since feedback is given during the task, participants slowly learn to associate the cues with the outcomes (Knowlton, Squire, \& Gluck, 1994). Again, both patient studies (Knowlton, Mangels, \& Squire, 1996; Perretta et al., 2005; Witt et al., 2006) and neuroimaging studies with healthy participants (Poldrack, Prabhakaran, Seger, \& Gabrieli, 1999; Seger \& Cincotta, 2005) have convincingly demonstrated the importance of the basal ganglia in probabilistic classification learning. Together, the large amount of literature on the involvement of the basal ganglia in different types of implicit learning processes underlines the importance of studying its possible involvement in implicit contextual learning. This is particularly interesting, since implicit contextual learning has different features than most of the implicit learning paradigms. That is, most of these paradigms are based on implicit motor learning (learning the associations between a cue and a motor response) or reward-related learning. In contrast, implicit contextual learning does not have such a motor or reward component and is purely based on visual learning, since the association between a target and the visual features of the environment is memorized. This would imply that the basal ganglia is not only involved in motor learning, but might have a more general role in implicit learning processes.
The aim of the current study was to investigate the role of the basal ganglia in implicit contextual learning by testing patients with Parkinson's disease. Parkinson's disease is a neurodegenerative disorder, in which reduced dopamine production due to neuronal loss in the substantia nigra leads to basal ganglia dysfunction. In the current study early-stage, non-demented Parkinson's disease patients were tested with a contextual cueing task, in order to define whether they are able to implicitly memorize repeated spatial context information.

\section{Methods}

2.1. Participants

Twenty-three patients with Parkinson's disease without dementia were examined. All patients had been admitted to the Department of Neurology in the University Hospital of Coimbra and were seen by an experienced neurologist (CJ, RA or AFG) to define the severity of the disease by using the Unified Parkinson's Disease Rating Scale (Fahn \& Elton, 1987) and the Hoehn and Yahr scale (Hoehn \& Yahr 1967). Additionally, 30 healthy age- and education matched controls were tested. None of the patients or control participants had a history of other neurological or psychiatric diseases. The study was approved by the medical ethics committee and written informed consent was obtained according to the declaration of Helsinki.

\subsection{Neuropsychological evaluation}

The Mini-Mental State Examination (Folstein, Folstein, \& McHugh, 1975) and the Hamilton Depression Rating Scale (Hamilton, 1960) were used to exclude patients with depression and signs of dementia respectively. Verbal intelligence was measured with the Vocabulary subtask of the Wechsler Adult Intelligence Scales (WAIS)-III. The Forward and Backward recall versions of the Corsi Block-Tapping task were used as a measure of spatial working memory. Handedness was defined by using a translated version of the Edinburgh Handedness Inventory (Oldfield, 1971). Informed consent was obtained according to the declaration of Helsinki and all procedures were approved by our local ethics committee.

\subsection{Apparatus and stimuli}

The software package Presentation (Neurobehavioral systems) was used to present a visual search task, in which 11 distractor stimuli $(L)$ and one target stimulus $(T)$ were presented each trial. The distractor stimuli were created in such a way as to make them look similar to the target stimulus (see Fig. 1). The stimulus size was $0.81^{\circ} \times 0.81^{\circ}$. The color of the stimuli was white and the background color was grey. Distractor stimuli could be rotated $0^{\circ}, 90^{\circ}, 180^{\circ}, 270^{\circ}$ and the target stimulus could be rotated $90^{\circ}$ or $270^{\circ}$.

\subsection{Procedure}

Participants were seated in a comfortable chair in a darkened room. After instructions were given, they were asked to place their chin in a chinrest that was positioned $50 \mathrm{~cm}$ from the computer screen. Twenty-four practice trials were given, including only new spatial configurations. If participants did not understand the task after the practice session, or were still making many errors, the practice trials were repeated. Participants were instructed to locate the target stimulus as quickly as possible, and indicate its orientation by pressing one of two buttons on the keyboard. The experiment included 16 blocks of trials, resulting in a total of 384 trials. Each block consisted of 24 trials, including 12 trials with a new configuration and 12 trials with a configuration that was repeated across blocks, appearing once in each block. Target position 
Table 1

Characteristics of the patient and control group.

\begin{tabular}{|c|c|c|c|c|}
\hline & \multicolumn{2}{|c|}{ Patients $N=23$} & \multicolumn{2}{|c|}{ Controls $N=30$} \\
\hline & Mean & SE & Mean & SE \\
\hline Age (years) & 58.0 & 1.4 & 55.2 & 1.4 \\
\hline $\operatorname{Sex}(m: f)$ & \multicolumn{2}{|c|}{$13: 10$} & \multicolumn{2}{|c|}{$11: 19$} \\
\hline Education (years) & 8.3 & 1.0 & 8.1 & 0.9 \\
\hline Age disease onset & 51.8 & 2.4 & - & - \\
\hline Disease duration & 6.8 & 1.3 & - & - \\
\hline UPDRS & 20.6 & 1.9 & - & - \\
\hline Hoehn and Yahr & 1.9 & 0.1 & - & - \\
\hline Hamilton & 3.5 & 0.6 & - & - \\
\hline MMSE & 29.3 & 0.3 & - & - \\
\hline Vocabulary (WAIS-III) & 35.4 & 2.9 & 36.0 & 2.6 \\
\hline \multicolumn{5}{|l|}{ Corsi Block-Tapping task } \\
\hline Forward & 34.3 & 1.5 & 44.3 & 2.8 \\
\hline Backward & 35.5 & 3.3 & 36.4 & 2.9 \\
\hline
\end{tabular}

Note: UPDRS = Unified Parkinson Disease Rating Scale, MMSE = Mini-Mental State Examination, WAIS-III = Wechsler Adult Intelligence Scales-III and SE=Standard Error.

in both repeated and new trials were randomly defined from the same set of positions. However, the positions of both the target and distractor stimuli were constant in the repeated configurations. The direction of rotation of the target stimulus was randomly defined, in order to prevent subjects of learning fixed stimulus-response associations. A fixation cross was presented before each trial during $500 \mathrm{~ms}$. A 10 second interval was given after each block so participants could take a short rest. For statistical analysis, the 16 blocks were collapsed into 4 epochs of 4 blocks each. Trials in which an error was made were excluded from analyses as well as trials with a response time that took longer than 2 SD above the individual mean.

After finishing the task, participants were asked to answer the following three questions: (1) 'Did you notice anything during the experiment?' (2) ‘Did you notice that some of the configurations were repeated?' (3) 'Did you try to remember the repeated configurations?' Finally, in order to verify whether context information was memorized implicitly, a Recognition memory control task was applied. This task consisted of 24 trials, including the 12 configurations that were repeated during the contextual cueing task and 12 new configurations. Repeated and new configurations were presented in a random order and participants were instructed to indicate whether the configuration was repeated during the experiment by pressing one of two keyboard buttons.

\section{Results}

\subsection{Neuropsychological tests}

Characteristics of the patient and control group are described in Table 1. No significant difference was found between the patient and control group for the Vocabulary test $[t(51)=0.1]$ and the backward recall condition of the Corsi Block-Tapping task $[t(50)=0.2]$. However, the control group did perform significantly better than the patient group for the forward recall condition of the Corsi BlockTapping task $[t(51)=2.9, p<0.01]$, indicating a working memory impairment in the Parkinson's disease patients. No difference was found between the patient and control group for age $[t(51)=1.4]$, education $[t(51)=0.1]$, or gender distribution $\left[\chi^{2}(1)=2.1\right]$.

\subsection{Contextual cueing task}

Both groups made very few errors (patients: 2.7\%; controls: $1.7 \%$ ) and few trials were excluded because of a delayed response (patients: 4.4\%; controls: 3.9\%). Overall, patients responded slower than the healthy controls $[F(1,51)=9.8, p<0.01]$.

We used the difference in response times between the new and repeated trials as a measure of contextual cueing. In line with previous patient studies (Chun \& Phelps, 1999; Manns \& Squire, 2001), we have only included the second part of the experiment for statistical analyses (Epoch 3-4). For the control participants an overall difference of $98.3 \mathrm{~ms}$ was found, whereas for the patients this difference was $-42.2 \mathrm{~ms}$. Statistical analyses showed a significant interaction between the variables Group (patients/controls)

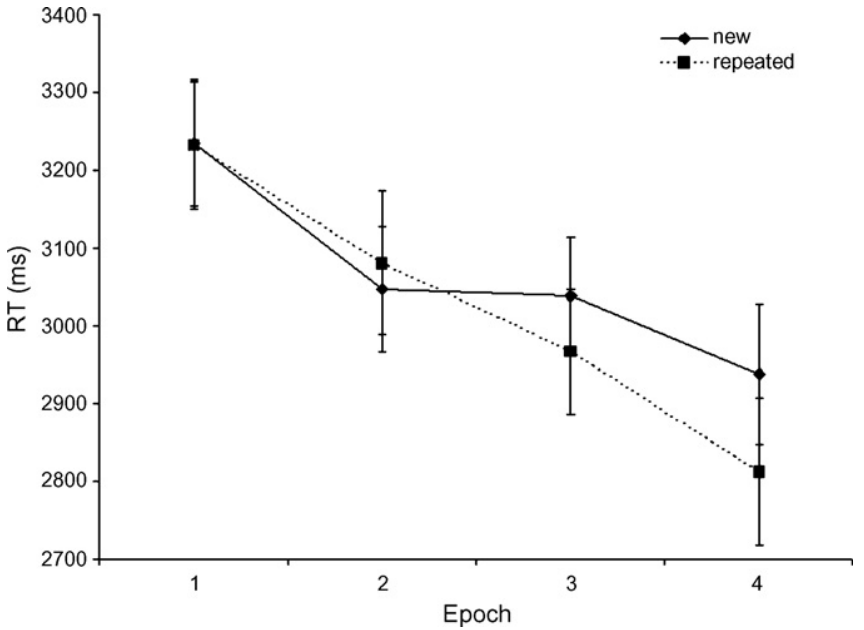

Fig. 2. Mean reaction times and standard errors of the control group for the Repeated and New trials separately as a function of Epoch (1-4).

and Configuration (repeated and new trials), indicating that the contextual cueing effect was significantly different for the patients than for the controls $[F(1,51)=5.9, p<0.05]$.

We also analyzed the response times of the patient and control group separately. For the healthy controls, a Repeated Measures analyses indicated that response times in the repeated trials were faster than in the new trials $[F(1,29)=5.6, p<0.05]$, reflecting implicit contextual learning (see Fig. 2). In contrast, no such learning effect was found for the Parkinson patients $[F(1,22)=1.4)]$ (see Fig. 3). This underlines the idea that damage to the basal ganglia due to Parkinson's disease impairs implicit contextual learning.

It could be argued that the Parkinson's patients group failed to show a contextual cueing effect since their overall response times were slower than the healthy control group. Therefore, we have transformed the response times into $Z$-scores based on each subjects' mean and standard deviation. These $Z$-scores were analyzed with the same Repeated Measures analyses as was previously used for the response times. Again, a significant interaction effect for Configuration and Group $[F(1,51)=5.9, p<0.05]$ was found. When the $Z$-scores were analyzed for the two groups separately we found a significant effect for Configuration for the control group $[F(1$, $29)=7.6, p<0.01]$, but not the Parkinson's patients $[F(1,22)=1.2]$. This indicates that after correcting for differences in response times

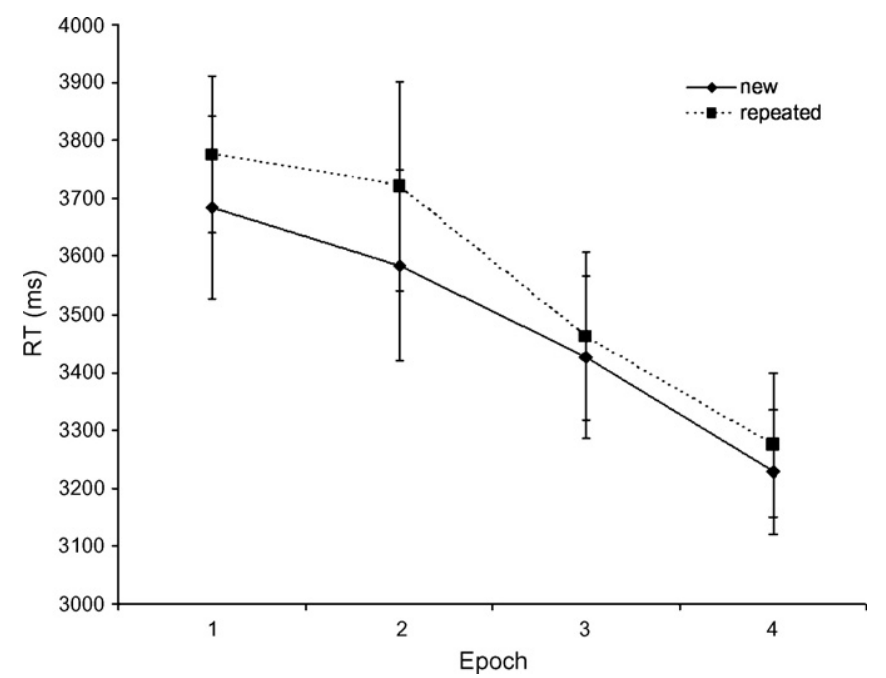

Fig. 3. Mean reaction times and standard errors of the Parkinson's disease patients for the Repeated and New trials separately as a function of Epoch (1-4). 
between the two groups, the Parkinson's patients still showed an impairment on the contextual cueing task.

Interestingly, Parkinson's disease does not seem to affect visuomotor learning. Indeed, during the visual search task the overall response times of the group decreased significantly $[F(3,153)=33.0$, $p<0.001$ ], regardless of Configuration. Most importantly, no interaction effect with Group $[F(3,153)=1.9]$ was found.

\subsection{Recognition memory control task}

In response to the questions asked before application of the Recognition memory task, none of the patients reported spontaneously to have noticed the repeated configurations. Seven out of 23 Parkinson's patients reported to have noticed some of the repeated trials when asked explicitly, but none of them reported trying to memorize the configurations. Although none of the controls reported spontaneously to have noticed the repetitions, 4 of the 30 control participants reported to have noticed the repetitions after explicitly being asked. One subject reported to have tried to remember the repetitions, although this subject had a performance of 12 correct responses on the Recognition memory task, which is at chance level (12 out of 24 trials correct).

On average, the control participants made $51.3 \%$ errors (5.8 repeated trials and 6.5 new trials). A one-sample $t$-test indicated no significant difference $[t(30)<0.8]$ with chance level (chance level $=12$ errors). Parkinson patients made $54 \%$ errors on average. A one-sample $t$-test indicated no difference between performance on the old and new trials and chance level $[t(21)<0.9]$.

\subsection{Correlation analysis}

Associated with Parkinson's disease is a working memory impairment revealed in our patients by a deficit on the forward recall condition of the Corsi Block-Tapping task, which reflects impaired spatial working memory. To exclude the possibility that the spatial working memory impairment is responsible for the contextual cueing impairment, a 2-tailed Pearson Correlation analysis was performed between the performance of the patients on the forward recall condition of the Corsi Block-Tapping task and the difference between the repeated and new trials of the last two epochs of the contextual cueing task, as a measurement of the performance on the contextual cueing task. No significant effect was found $(r=0.04)$, suggesting that these two types of impairment are not related.

\section{Discussion}

The current study was aimed at investigating implicit contextual learning in patients with damage to the basal ganglia due to Parkinson's disease. Therefore, a visual search task was used, in which half of the trials included spatial configurations that were repeated throughout the experiment. Healthy control participants were able to benefit from the repeated trials, as was shown by the faster response times when the context information was repeated in comparison to the trials with new configurations. This contextual cueing effect is due to a truly implicit learning mechanism, as is reflected by the chance level performance on the Recognition memory task. In contrast, no difference in response times between the repeated and new trials was found for the patients with Parkinson's disease, indicating an implicit contextual cueing deficit. Importantly, the contextual cueing effect of the control group differed significantly from the Parkinson's patients. The finding that Parkinson patients failed to benefit from a repeated context suggests an important role for the basal ganglia in implicit contextual learning. This extends previous findings that have underlined the role of the basal ganglia in implicit learning processes (Daselaar et al., 2003;
Doyon et al., 2003; Kim et al., 2004; Knowlton et al., 1996, 1994; Perretta et al., 2005; Poldrack et al., 1999; Rauch et al., 1997; Seger \& Cincotta, 2005; Wilkinson \& Jahanshahi, 2007; Witt et al., 2006). An important difference between the previously studied implicit learning tasks and the currently used task is the fact that implicit contextual learning is not based on the association between perception and motor responses. This suggests that the basal ganglia is not only involved in motor learning, but also has a role in purely visual implicit learning mechanisms. These results further extend the conclusions of Castelo-Branco et al. (2009) who showed that the basal ganglia are important for visual functions.

Previous studies aimed at defining the neural correlates of implicit contextual cueing have been limited to the MTL (Chun \& Phelps, 1999; Greene et al., 2007; Manns \& Squire, 2001). The idea that the MTL and basal ganglia are involved in implicit contextual learning is interesting and in line with recent studies that have shown involvement of both brain areas in a single learning process. Poldrack et al. (2001) used functional neuroimaging to show that classification learning depends on the MTL during early learning phases, while this dependence rapidly declines with training. In contrast, the caudate nucleus (which is a part of the basal ganglia) is inactive in initial stages, and becomes active in later stages. Poldrack et al. (2001) suggest that the MTL might acquire flexible, relational knowledge, whereas the striatum might be involved in creating inflexible stimulus-response associations, reflecting declarative and nondeclarative memory respectively. In the case of implicit contextual cueing, the MTL might be involved in an early learning phase, in which the relations between the target and context information are learned and the basal ganglia becomes involved when visual search is based on learned stimulus-response associations. However, it is not clear whether this would necessarily reflect declarative and nondeclarative learning respectively, since no direct evidence was found of declarative memory during implicit contextual learning. It should be noted that the finding of MTL involvement in implicit contextual cueing does raise the question whether the neural correlates of declarative and nondeclarative memory are strictly based on the MTL and the basal ganglia respectively. Disentangling the exact role of the MTL and the basal ganglia in implicit contextual learning is therefore not only important for our understanding of the neural correlates of this learning mechanism, but can also help to understand the mechanism underlying the MTL and basal ganglia interaction.

It should be noted that Parkinson patients show normal visuospatial skill learning since the overall response time decreases during the experiment to the same extent for the patients and controls. This is important, since it indicates that implicit learning effects can be measured in these subjects, in spite of motor deficits leading to decreased response times.

Parkinson disease patients were significantly impaired on the forward recall condition of the Corsi Block-Tapping task, supporting previous findings of a spatial working memory impairment (Kemps, Szmalec, Vandierendonck, \& Crevits, 2005; Stoffers, Berendse, Deijen, \& Wolters, 2003). Interestingly, performance on the backward recall condition of the Corsi Block-Tapping task is normal. In contrast to the forward recall condition, the backward recall condition requires subjects to reorganize the spatial information, thus making it more dependent on executive functioning. It should be noted that the contextual cueing impairment that is found in the current study is most likely not due to this spatial working memory deficit, as no correlation was found. Although spatial working memory might be important for making a visual search in general and therefore partly explain the general effect of slower response times, this accounts for the new and repeated trials to the same extent.

In sum, the current study has shown that Parkinson's disease patients with damage to the basal ganglia due to dopamine deple- 
tion are impaired on an implicit contextual learning task. This suggests that the basal ganglia have an important role in acquiring spatial context information through an implicit memory process. These results give a new direction in the search for the neural correlates of implicit contextual learning by emphasizing the role of the basal ganglia. Future studies should be aimed at defining the relative roles of the MTL and basal ganglia in implicit contextual learning.

\section{Acknowledgements}

This research was supported by a grant from the BIAL Foundation (no. 73/06) and the FCT (POCI_SAU-NEU_60281_2004). Marieke van Asselen was supported by an FCT grant (SFRH/BPD/22088/2005).

\section{References}

Castelo-Branco, M., Mendes, M., Silva, F., Massano, J., Januário, G., Januário, C., \& Freire, A. (2009). Motion integration deficits are independent of magnocellular impairment in Parkinson's disease. Neuropsychologia, 47, 314-320.

Chun, M. M., \& Jiang, Y. (1998). Contextual cueing: Implicit learning and memory of visual context guides spatial attention. Cognitive Psychology, 36, 28-71.

Chun, M. M. (2000). Contextual cueing of visual attention. Trends in Cognitive Sciences, $4(5), 170-178$.

Chun, M. M., \& Jiang, Y. (1999). Top-down attentional guidance based on implicit learning of visual covariation. Psychological Science, 10(4), 360-365.

Chun, M. M., \& Jiang, Y. (2003). Implicit, long-term spatial contextual memory. Journal of Experimental Psychology: Learning, Memory, and Cognition, 2, 224-234.

Chun, M. M., \& Phelps, E. A. (1999). Memory deficits for implicit contextual information in amnesic subjects with hippocampal damage. Nature Neuroscience, 2(9), 844-847.

Daselaar, S. M., Rombouts, S. A. R. B., Veltman, D. J., Raaijmakers, J. G. W., \& Jonker, C. (2003). Similar network activated by young and old adults during the acquisition of a motor sequence. Neurbiology of Aging, 24, 1013-1019.

Doyon, J., Penhune, V., \& Ungerleider, L. G. (2003). Distinct contribution of the corticostriatal and cortico-cerebellar systems to motor skill learning. Neuropsychologia $41,252-262$.

Fahn, S., \& Elton, R. L. (1987). Unified Parkinson's disease rating scale. In S. Fahn, C D. Marsden, D. B. Calne, \& M. Goldstein (Eds.), Recent developments in Parkinson's disease (pp. 153-163). Florham Park, NJ: MacMillan Healthcare Information.

Folstein, M. F., Folstein, S. E., \& McHugh, P. R. (1975). 'Minimental state': A practical method for grading the cognitive state of patients for the clinician. Journal of Psychiatric Research, 12, 189-198.

Greene, J. A., Gross, W. L., Elsinger, C. L. \& Rao, S. M. (2007). Hippocampal differentiation without recognition: An fMRI analysis of the contextual cueing task. Learning \& Memory, 10, 548-553.
Hamilton, M. (1960). A rating scale for depression. Journal of Neurology, Neurosurgery, and Psychiatry, 23, 56-62.

Hoehn, M., \& Yahr, M. (1967). Parkinsonism: Onset, progression and mortality. Neurology, 17(5), 427-442.

Jiang, Y., \& Leung, A. W. (2005). Implicit learning of ignored visual context. Psychonomic Bulletin \& Review, 12, 100-106.

Jiang, Y., Song, J. H., \& Rigas, A. (2005). High-capacity spatial contextual memory. Psychonomic Bulletin E Review, 12, 524-529.

Kemps, E., Szmalec, A., Vandierendonck, A., \& Crevits, L. (2005). Visuo-spatial processing in Parkinson's disease: Evidence for diminished visuo-spatial sketch pad and central executive resources. Parkinsonism E Related Disorders, 11(3), 181-186.

Kim, J. S., Reading, S. A., Brashers-Krug, T., Calhoun, V. D., Ross, C. A., \& Pearlson, G. D. (2004). Functional MRI study of a serial reaction time task in Huntington's disease. Psychiatry Research, 131(1), 23-30.

Knowlton, B. J., Mangels, J. A., \& Squire, L. R. (1996). A neostriatal habit learning system in humans. Science, 273(5280), 1399-1402.

Knowlton, B. J., Squire, L. R., \& Gluck, M. A. (1994). Probabilistic classification learning in amnesia. Learning and Memory, 1(2), 106-120.

Manns, J. R., \& Squire, L. R. (2001). Perceptual learning, awareness, and the hippocampus. Hippocampus, $11,776-782$.

Oldfield, R. C. (1971). The assessment and analysis of handedness: The Edinburgh inventory. Neuropsychologia, 9(1), 97-113.

Olson, I. R., \& Chun, M. M. (2002). Perceptual constraints on implicit learning of spatial context. Visual Cognition 9, 273-302.

Perretta, J. G., Pari, G., \& Beninger, R. J. (2005). Effects of Parkinson Disease on Two Putative Nondeclarative Learning Tasks. Cognitive and Behavioral Neurology 18(4), 185-192.

Peterson, M. S., \& Kramer, A. F. (2001). Attentional guidance of the eyes by contextua information and abrupt onsets. Perception \& Psychophysics, 63(7), 1239-1249.

Poldrack, R. A., Clark, J., Paré-Blagoev, E. J., Shohamy, D., Creso Moyano, J., Myers, C., et al. (2001). Interactive memory systems in the human brain. Nature, 414 546-550.

Poldrack, R. A., Prabhakaran, V., Seger, C. A., \& Gabrieli, J. D. (1999). Striatal activation during acquisition of a cognitive skill. Neuropsychology, 13(4), 564-574.

Rauch, S. L. R., Whalen, P. J., Savage, C. R., Curran, T., Kendrick, A., Brown, H. D., et al. (1997). Striatal recruitment during an implicit sequence learning task as measured by functional magnetic resonance imaging. Human Brain Mapping, 5 124-132.

Seger, C. A., \& Cincotta, C. M. (2005). The roles of the caudate nucleus in human classification learning. Journal of Neuroscience, 16(25 (11)), 2941-2951.

Stoffers, D., Berendse, H. W., Deijen, J. B., \& Wolters, E. C. (2003). Deficits on Cors block-tapping task in early stage Parkinson's disease. Parkinsonism and Related Disorders, 10(2), 107-111

Tseng, Y. C., \& Li, C. S. (2004). Oculomotor correlates of context-guided learning in visual search. Perception \& Psychophysics, 66, 1363-1378.

Wilkinson, L., \& Jahanshahi, M. (2007). The striatum and probabilistic implicit sequence learning. Brain Research, 1137, 117-130.

Witt, K., Daniels, C., Daniel, V., Schmitt-Eliassen, J., Volkmann, J., \& Deuschl, G. (2006). Patients with Parkinson's disease learn to control complex systems-An indication for intact implicit cognitive skill learning. Neuropsychologia, 44, 2445-2451. 